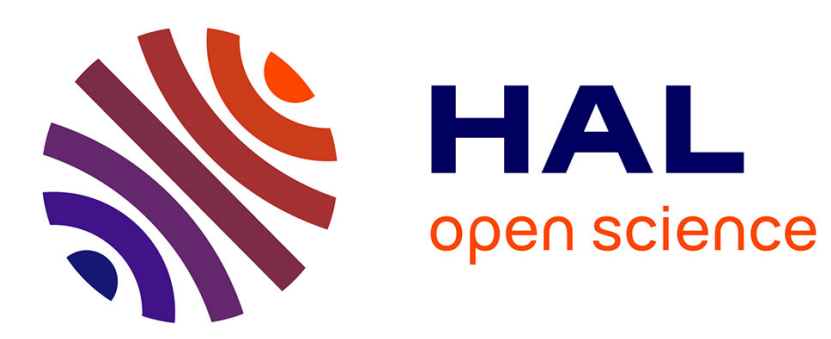

\title{
Domain Decomposition for Computing Extremely Low Frequency Induced Current in the Human Body
}

Ronan Perrussel, Damien Voyer, Laurent Nicolas, Riccardo Scorretti, Noël Burais

\section{- To cite this version:}

Ronan Perrussel, Damien Voyer, Laurent Nicolas, Riccardo Scorretti, Noël Burais. Domain Decomposition for Computing Extremely Low Frequency Induced Current in the Human Body. IEEE Transactions on Magnetics, 2011, 47 (5), pp.886 - 889. 10.1109/TMAG.2010.2076273 . hal-00643992

\section{HAL Id: hal-00643992 \\ https://hal.science/hal-00643992}

Submitted on 23 Nov 2011

HAL is a multi-disciplinary open access archive for the deposit and dissemination of scientific research documents, whether they are published or not. The documents may come from teaching and research institutions in France or abroad, or from public or private research centers.
L'archive ouverte pluridisciplinaire HAL, est destinée au dépôt et à la diffusion de documents scientifiques de niveau recherche, publiés ou non, émanant des établissements d'enseignement et de recherche français ou étrangers, des laboratoires publics ou privés. 


\title{
Domain Decomposition for Computing Extremely Low Frequency Induced Current in the Human Body
}

\author{
Ronan Perrussel ${ }^{1}$, Damien Voyer ${ }^{1}$, Laurent Nicolas ${ }^{1}$, Riccardo Scorretti $^{2}$, and Nöel Burais ${ }^{2}$ \\ ${ }^{1}$ Laboratoire Ampère UMR 5005 CNRS, Ecole Centrale de Lyon, Ecully 69314, France \\ ${ }^{2}$ Laboratoire Ampère UMR 5005 CNRS, Université Lyon 1, Villeurbanne 69622, France
}

\begin{abstract}
Computation of electromagnetic fields in high resolution computational phantoms requires solving large linear systems. We present an application of Schwarz preconditioners with Krylov subspace methods for computing extremely low frequency induced fields in a phantom issued from the Visible Human.
\end{abstract}

Index Terms-Extremely low frequency, finite-element methods, numerical dosimetry.

\section{INTRODUCTION}

$\mathbf{N}$ UMERICAL dosimetry of electromagnetic fields into the human body is important for assessing safety of devices which generate strong fields (such as magnetic resonance imagery systems or welding industry). First computations of induced electromagnetic fields into the human body date at $80 \mathrm{~s}$ [1], but it is mainly during nineties that important advances have been made by the scientific community, among others by Stuchly et al. [2]-[4]. At the same time, the milestone work of Gabriel et al. brought a large amount of key data on the electrical properties of tissues [5]-[7].

Most of the existing literature is based on computations using the finite-difference (FD) method, or some closely related variants like the impedance method (IM). All these methods have the advantage that a computational phantom can easily be obtained on a Cartesian grid from segmented images of the human body. However the price to pay is that this approach requires a huge number of degrees-of-freedom (DOFs). When an explicit temporal scheme is adopted, there is no linear system to invert: time iterations are taken on until the steady state is reached with a "sufficient" accuracy. As the problem is linear, frequency scaling techniques are used to avoid instabilities due to large time steps [8]. More recently, the finite-integration method (FIT) has been used; in this case, the human body is discretized over a structured mesh, and therefore a huge number of DOFs is also required [9].

The finite-element method (FEM) has been seldom used to solve these kind of problems, mainly because of the difficulty to obtain an unstructured and adaptive mesh of the computational phantom-which is key for taking advantage of the features of this method. However, recently methods for building such a mesh have been proposed [10], making possible to perform more efficient computations based on FEM.

The purpose of this paper is to show how domain decomposition preconditioners [11] combined with Krylov subspace solvers [12] can be used to solve this kind of linear systems.

Manuscript received May 05, 2010; revised July 05, 2010; accepted August 23, 2010. Date of current version April 22, 2011. Corresponding author: R. Scorretti (e-mail: riccardo.scorretti@univ-lyon1.fr).

Color versions of one or more of the figures in this paper are available online at http://ieeexplore.ieee.org.

Digital Object Identifier 10.1109/TMAG.2010.2076273
The presented algorithms enable to improve the convergence for solving the systems, and are naturally well suited for distributed parallel computing.

\section{II. $\phi-\mathbf{a}$ FORMULATION}

Due to the particular features of the living tissues, specific formulations have been developed [13]. Consider a magnetic vector potential source $\mathbf{a}$ such that $\mathbf{b}=\operatorname{curl} \mathbf{a}$. It is assumed that $\mathbf{b}$ is not modified by the presence of the human body (domain $\Omega$ ); therefore a is known a priori. From Faraday's law $\operatorname{curl} \mathbf{e}=-\partial_{t} \mathbf{b}$ one obtains $\mathbf{e}=-\partial_{t} \mathbf{a}-\operatorname{grad} \phi$, where the scalar potential $\phi \in \mathrm{H}^{1}(\Omega)$ is the unknown. Displacement currents are neglected; from Ampère's equation curl $\mathbf{h}=\mathbf{j}$ one obtains the current continuity equation $\operatorname{div} \mathbf{j}=0$. Supplemented by Ohm's law $\mathbf{j}=\sigma \mathbf{e}$ and imposed weakly it gives

$$
\begin{aligned}
& \text { Find } \phi \text { in } \mathrm{H}^{1}(\Omega) \text { such that: } \\
& (\sigma \operatorname{grad} \phi, \operatorname{grad} w)+\left(\sigma \partial_{t} \mathbf{a}, \operatorname{grad} w\right)=0, \quad \forall w \in \mathrm{H}^{1}(\Omega) .(1)
\end{aligned}
$$

At the discrete level $\phi$ is expanded on nodal functions, hence a gauge is simply fixed by imposing the value of $\phi$ in a node. This formulation leads to a large sparse linear system $\mathbf{A x}=\mathbf{f}$ which is solved by using a Krylov subspace solver.

\section{SCHWARZ PRECONDITIONERS}

In Schwarz preconditioners, the computational domain $\Omega$ is decomposed in several overlapping subdomains $\Omega_{1, \ldots, N}$. Each subdomain is discretized on a mesh $\mathcal{M}_{i}$, which may eventually be a subset of a global mesh $\mathcal{M}$ : in this case the nodes on the overlapping regions are shared between several partial meshes (matching meshes). The formulation is assembled over each subdomain $\Omega_{i}$; hence $N$ linear systems $\mathbf{A}_{i} \mathbf{x}_{i}=\mathbf{f}_{i}$ are generated. At each step of the algorithm, the residual is restricted to the interior $\overline{\Omega_{i}} \backslash\left(\partial \Omega_{i} \backslash \partial \Omega\right)$ of each subdomain $\Omega_{i}$, a local correction is computed and projected back to the global domain. All Schwarz preconditioners are founded on this general idea. Many variants of this class of preconditioners exist, which differ mainly in the number of levels and in the order the different corrections are computed. 


\section{A. Restriction/Prolongation Operators}

A key point is that each local vector $\mathbf{x}_{i}$ can be easily obtained from the global vector $\mathbf{x}$ through a linear restriction operator of matrix $\mathbf{R}_{i}$

$$
\mathbf{x}_{i}=\mathbf{R}_{i} \mathbf{x}
$$

In the same way, a linear prolongation operator of matrix $\mathbf{P}_{i}$ allows to obtain the global contribution which correspond to each local vector, so that

$$
\mathbf{A}_{i}=\mathbf{R}_{i} \mathbf{A} \mathbf{P}_{i}
$$

In the case of matching meshes, $\mathbf{R}_{i}$ is a permutation of the matrix ( $\left.\begin{array}{ll}\mathbf{I} & 0\end{array}\right)$, and it can be shown that $\mathbf{P}_{i}$ is simply the transposed matrix of $\mathbf{R}_{i}$. In the general case of non-matching meshes, each term of the local vector is computed from the global one by using a first-order finite-element interpolation. The prolongation matrix $\mathbf{P}_{i}$ is obtained by transposing $\mathbf{R}_{i}$ and normalizing the sum of the terms over each row to one: therefore the prolongation of the local correction over a node of $\mathcal{M}$ is computed by averaging its (local) value over the neighbor nodes of $\mathcal{M}_{i}$. We stress that this is not the unique way to perform the restriction/prolongation: other methods can be used-for instance in [14] is described an approach based on aggregation of neighbor nodes.

\section{Algorithm 1 One-level additive Schwarz preconditioner}

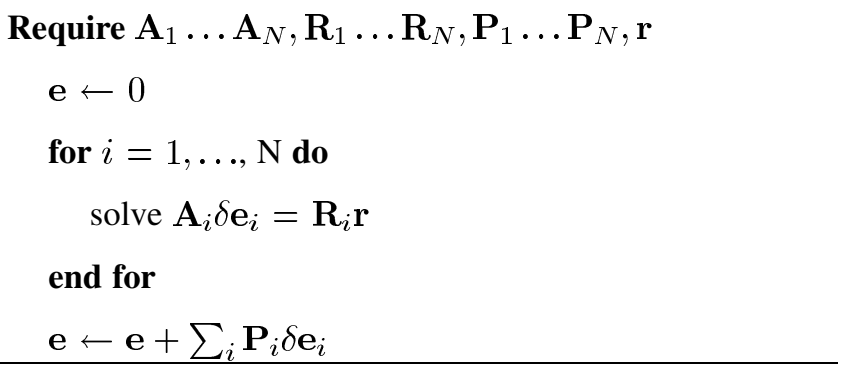

\section{B. One- and Two-Levels Methods}

In one-level methods, a local correction is computed on each subdomain from the local restriction of the residual $\mathbf{r}$. There exist many variants of this method, one of which is Algorithm 1. One observes that the residual $\mathbf{r}$ is not updated, therefore all subdomains can be processed in parallel—the corrections $\delta \mathbf{e}_{i}$ being accumulated together afterward.

The link between the subdomains is uniquely ensured by the overlap, which is usually limited to a few layers of elements. One observes that: 1) the number of iterations increases with the number of subdomains and 2) the less the size of the overlap, the more iterations are required. This fact can be explained heuristically by arguing that the information needs to "travel" through all subdomains before reaching the other extremity of the whole domain: therefore, the long-range (i.e., low-frequency) terms of the error require many iterations for being corrected [11].

A better behavior can be obtained by a two-level method: in this case, an additional correction is computed by using the same strategy (i.e., restrict/solve/project back) with a global coarse mesh $\mathcal{M}_{c}$ in order to correct the long-range terms of the error. Algorithm 2 is obtained by adding a correction in a multiplicative way to Algorithm 1 (it is called a hybrid version in [15]). This correction does not change significantly the computational time between both algorithms. $\mathbf{R}_{c}$ and $\mathbf{P}_{c}$ are the restriction/ prolongation matrix for the coarse mesh computed as described beforehand.

Algorithm 2 Two-level hybrid Schwarz preconditioner

Require $\mathbf{A}_{1} \ldots \mathbf{A}_{N}, \mathbf{R}_{1} \ldots \mathbf{R}_{N}, \mathbf{P}_{1} \ldots \mathbf{P}_{N}, \mathbf{R}_{c}, \mathbf{P}_{c}, \mathbf{r}$

solve $\mathbf{R}_{c} \mathbf{A} \mathbf{P}_{c} \delta \mathbf{e}_{c}=\mathbf{R}_{c} \mathbf{r}$

$\mathbf{e} \leftarrow \mathbf{P}_{c} \delta \mathbf{e}_{c}$

for $i=1, \ldots, N$ do

solve $\mathbf{A}_{i} \delta \mathbf{e}_{i}=\mathbf{R}_{i} \mathbf{r}$

end for

$\mathbf{e} \leftarrow \mathbf{e}+\sum_{i} \mathbf{P}_{i} \delta \mathbf{e}_{i}$

\section{Implementation}

Both algorithms 1 and 2 have been implemented by a sequential program written in MATLAB. As the matrix $\mathbf{A}$ is symmetric positive definite, we choose the Conjugate Gradient (CG) as the Krylov subspace method. Each subdomain linear system is solved by using a direct solver. Indeed, the theory for Krylov subspace methods requires that the preconditioner is a linear operator [11]. Some local approximate solvers do not enable us to satisfy this linearity assumption and should lead us to consider flexible variant of CG [16]. We tried several approximate solvers, but except some small academical problems, a poor convergence has been observed.

\section{VALIDATION AND PRELIMINARY Tests}

We performed some tests with an academical benchmark (exposure of a homogeneous spheroid to a uniform magnetic field-Fig. 1) by using one and two-level methods. $\mathrm{CG}$ is stopped when the residual is reduced by a factor $10^{6}$ with respect to its initial value. The theoretical values of the average and maximum electric field induced by the extremely low frequency magnetic field are [17]

$$
\begin{aligned}
E_{\max } & =\omega\left\|\mathbf{b}_{0}\right\| \frac{a^{2} b}{a^{2}+b^{2}} \\
E_{\mathrm{avg}} & =\frac{3}{8} \mathrm{E}\left(1-b^{2} / a^{2}\right) E_{\max }
\end{aligned}
$$

where $\mathrm{E}(m)=\int_{0}^{\pi / 2} \sqrt{1-m \sin ^{2} \theta} d \theta$ is the complete elliptic integral of second kind, $a$ and $b$ are for the major and minor axis, respectively. The comparison between the theoretical and numerical values of the electric field are reported in Table I for $a=60 \mathrm{~cm}, b=30 \mathrm{~cm},\left\|\mathbf{b}_{0}\right\|=500 \mu \mathrm{T}$ and $f=50 \mathrm{~Hz}$ (Algorithms 1 and 2 provide the same results). The large error on $E_{\max }$ is due to the artifacts which are clearly visible at the surface of the ellipsoide-the $99 \%$ percentile values $(99 \% \mathrm{E})$ being much better estimates of the true $E_{\max }$. The results of Table II show that as the size of the problem increases, the two- 

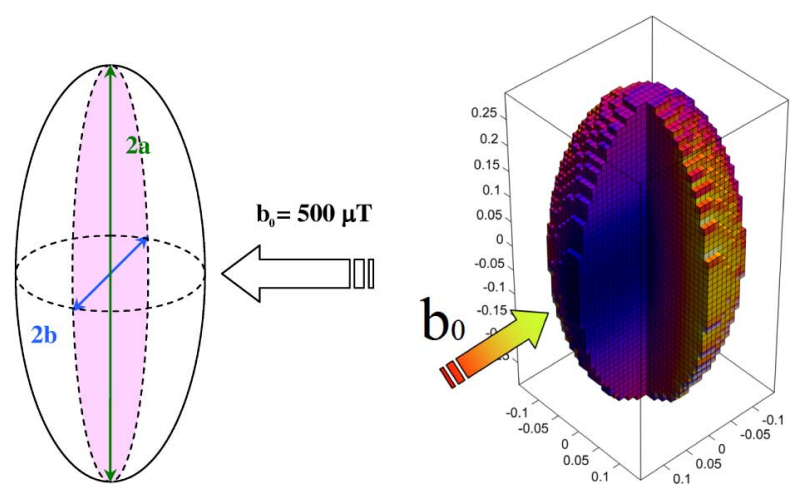

Fig. 1. Prolate ellipsoid exposed to a field $\left\|\mathbf{b}_{0}\right\|=500 \mu \mathrm{T}, f=50 \mathrm{~Hz}$.

TABLE I

ELECTRIC FIELD $(\mathrm{MV} / \mathrm{M})$ FOR A $120 \times 60 \mathrm{~cm}$ ELLIPSOID. \# DENOTES "NUMBER OF"

\begin{tabular}{ll|lll}
\hline \hline & \# DOFs & Avg E & Max E & $99 \% \mathrm{E}$ \\
\hline$B x=500 \mu \mathrm{T}$ & $30 \mathrm{~K}$ & 17.3 & 53.0 & 37.1 \\
& $120 \mathrm{~K}$ & 17.4 & 67.2 & 39.0 \\
& Analytic & 17.1 & 37.7 & $*$ \\
\hline$B z=500 \mu \mathrm{T}$ & $30 \mathrm{~K}$ & 13.9 & 28.2 & 24.9 \\
& $120 \mathrm{~K}$ & 13.9 & 30.1 & 24.1 \\
& Analytic & 13.9 & 23.6 & $*$ \\
\hline \hline
\end{tabular}

TABLE II

NUMBER OF ITERATIONS FOR THE BENCHMARK. \# DENOTES "NUMBER OF"

\begin{tabular}{cllcc}
\hline \hline \# subdomains & \multicolumn{2}{l}{ resolution (\# DOFs) } & \# iter. (1-lev) & \# iter. (2-lev) \\
\hline $2 \times 2 \times 2$ & $16 \mathrm{~mm}$ & $(30 \mathrm{~K})$ & 12 & 7 \\
$4 \times 4 \times 8$ & $16 \mathrm{~mm}$ & $(30 \mathrm{~K})$ & 14 & 6 \\
$8 \times 8 \times 16$ & $16 \mathrm{~mm}$ & $(30 \mathrm{~K})$ & 15 & 6 \\
$2 \times 2 \times 4$ & $8 \mathrm{~mm}$ & $(230 \mathrm{~K})$ & 21 & 6 \\
$4 \times 2 \times 6$ & $5 \mathrm{~mm}$ & $(1 \mathrm{M})$ & 36 & 9 \\
\hline \hline
\end{tabular}

level method (2-lev) outperforms the one-level method (1-lev).

\section{Comparison With Available Data}

We simulated the exposure of the human body to a $60 \mathrm{~Hz}$, uniform $1 \mathrm{mT}$ back-to-front magnetic field, and compared the results with the values found in literature [3], [18], [19]. The computational phantom is based on the Visible Human (see Fig. 2). It is discretized with a resolution of $3 \mathrm{~mm}(4.2 \mathrm{M} \mathrm{DOFs})$ using a structured hexahedral mesh, and decomposed into a grid of $5 \times 3 \times 15$ subdomains, with an overlap of five elements. The coarse mesh has a resolution of $16 \mathrm{~mm}$. The computation has been performed with the two-level preconditioner in 12 iterations.

The computed average value of the electric field over each organ are reported in the last column of Table III; this table collects the available data obtained with different computational phantoms (UT $=$ Zubal (Univ. Utah) [3], UVic = Univ. Victoria [3], [18], $\mathrm{AF}=$ Brook Air Force [18], ZOL [19]) and methods (IM, SPFD $=$ Scalar Potential FD, TDFD = Time Domain FD, FEM). In [3] the average value is based of punctual values, whereas in [18] the field is previously averaged over $1 \mathrm{~cm}^{2}$; this may contribute to explain the difference between the reported values. The maximum values of the electric field have not been

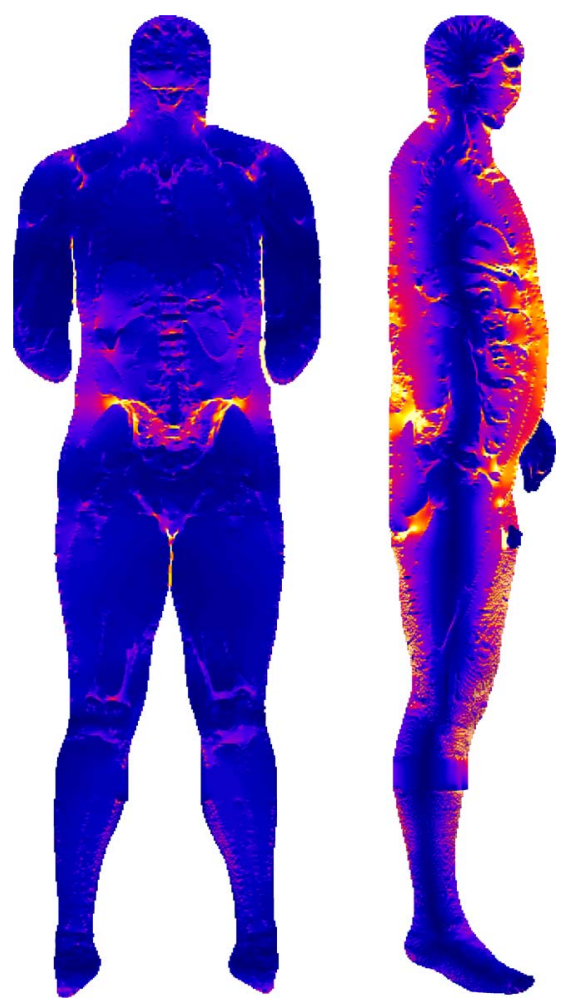

Fig. 2. Electric field plotted in a section of the body.

compared, on one hand because they are much more sensitive to numerical errors [20], and on the other hand because they are not always available. The statistic used for comparing the different results is the following:

$$
D=\frac{\sigma}{\bar{x}}=\frac{\sqrt{\frac{1}{n-1} \sum_{i}^{n}\left(x_{i}-\bar{x}\right)^{2}}}{\frac{1}{n} \sum_{i}^{n} x_{i}} \times 100 \%
$$

where $x_{i}$ is the average value of the electric field on a given organ for the $i$ th column of Table III-that is the $i$ th method-and $\bar{x}$ is the average of $x_{i}$. The smaller is $D$, the better is the coherence of data. Both the phantoms AF, ZOL and the one used in the present work are based on the Visible Human; therefore differences cannot be explained by morphological differences, but only by numerical errors.

When all the available data is considered, the average value of $D$ is $52 \%$. Comfortably, one observes that if only the values based on UVic are considered, the average value of $D$ decreases to $11 \%$. Similarly, if only the values based on the Visible Human are considered the average value of $D$ is $20 \%$ (it decreases to $15 \%$ if ZOL-i.e., column 7-is removed from Table III). Only the organs for which data are available for all the phantoms are considered. One observes an abnormally low value of the electric field in testis in our computation; at present time we cannot explain this value, which could probably be attributed to some problems in the decimation of the segmented images.

\section{CONCLUSION}

This work presents an application of domain decomposition to numerical dosimetry. Even if the implementation is still not 
TABLE III

AVERAge Electric Field (MV/M) ObTAINED IN THIS Work (LAST COLUMN) AND IN Literature

\begin{tabular}{|c|c|c|c|c|c|c|c|}
\hline Organ & $\begin{array}{c}\text { UT [3] } \\
\text { IM }\end{array}$ & $\begin{array}{c}\text { UVic }(3.6 \mathrm{~mm})[3] \\
\text { FDTD }\end{array}$ & $\begin{array}{c}\text { UVic }(7.2 \mathrm{~mm})[3] \\
\text { FDTD }\end{array}$ & $\begin{array}{c}\text { UVic }(3.6 \mathrm{~mm})[18] \\
\operatorname{SPFD}^{(c)}\end{array}$ & $\begin{array}{c}\mathrm{AF}(2 \mathrm{~mm})[18] \\
\operatorname{SPFD}^{(c)}\end{array}$ & $\begin{array}{l}\text { ZOL [19] } \\
\mathrm{FEM}^{(d)}\end{array}$ & $\begin{array}{c}\text { Our results } \\
\text { FEM }\end{array}$ \\
\hline Bladder & 18.9 & 8.98 & 9.8 & $*$ & * & * & 16.1 \\
\hline $\operatorname{Brain}^{(a)}$ & 11.5 & 10.6 & 11.5 & 8.82 & 10.3 & 17.5 & 11.2 \\
\hline Cerebral spinal fluid & 2.8 & 5.1 & 4.3 & 4.28 & 9.15 & $*$ & 4.4 \\
\hline Eye aqueous humor & 2.1 & 3.5 & 3.6 & * & $*$ & * & 1.46 \\
\hline Heart & 12.4 & 13.5 & 17.8 & 14.4 & 16.7 & 15.9 & 11.9 \\
\hline Intestine (bowel) & 16.5 & 17.5 & 18.2 & $*$ & $*$ & $*$ & 15.9 \\
\hline Kidneys & 20.2 & 25.2 & 23.3 & 20.6 & 20.4 & 30.8 & 26.7 \\
\hline Liver & 25.8 & 34.4 & 26.4 & * & * & * & 35.3 \\
\hline $\operatorname{Lungs}^{(a)}$ & 28.8 & 20.8 & 22.0 & 18.9 & 20.7 & 21.6 & 17.6 \\
\hline Pancreas & 18.0 & 7.9 & 11.4 & * & * & $*$ & 6.38 \\
\hline Prostate $^{(b)}$ & 21.7 & 17.0 & 17.4 & * & * & * & * \\
\hline $\operatorname{Stomach}^{(a)}$ & 11.2 & 10.4 & 13.9 & * & * & $*$ & 12.8 \\
\hline Testis & 34.7 & 14.8 & 18.7 & 13.0 & 14.2 & $*$ & 3.9 \\
\hline
\end{tabular}

(a) In [3] there is no distinction between white and gray matter; in this one and similar situations (inner and outer lung, small and large bowel) the averaged value is reported. ${ }^{(b)}$ In some phantoms the prostate is not identified. ${ }^{(c)}$ Averaged value over $1 \mathrm{~cm}^{2}{ }^{2}(d)$ Non conform tetrahedral mesh.

optimized (in particular, the code is not parallelized), the feasibility of this approach is demonstrated. Future works will be oriented towards: 1) a true parallel implementation; 2 ) the use of non structured mesh; 3 ) the evaluation of optimized Schwarz algorithm [21]. The comparison with data found in literature suggests that the morphological differences are the main factor for explaining difference in numerical dosimetry. Also, it is shown how even with a rather coarse phantom like ZOL it is possible to obtain at least the correct order of magnitude of the average fields (with differences in magnitude of the order of 50\%) -with a computation time of a few minutes. The availability of more accurate computational phantoms [22] should allows to better quantify the different uncertainties and their role on the accuracy of the results.

\section{ACKNOWLEDGMENT}

The authors would like to thank RTE/EDF for their financial support. One of the authors, R. Scorretti, would like to thank the University of Liège for their very kind hospitality during his visit.

\section{REFERENCES}

[1] O. P. Gandhi, J. F. Deford, and H. Kanai, "Impedence method for calculation of power deposition patterns in magnetically induced hyperthermia," IEEE Trans. Biomed. Eng., vol. 31, no. 10, pp. 644-651, Oct. 1984.

[2] T. W. Dawson, K. Caputa, and M. A. Stuchly, "Influence of human model resolution on computed currents induced in organs by $60-\mathrm{Hz}$ magnetic fields," Bioelectromagn., vol. 18, no. 7, pp. 478-490, 1998.

[3] M. A. Stuchly and O. P. Gandhi, "Inter-laboratory comparison of numerical dosimetry for human exposure to $60 \mathrm{~Hz}$ electric and magnetic fields," Bioelectromagn., vol. 21, no. 3, pp. 167-174, 2000.

[4] P. J. Dimbylow, "Current densities in a $2 \mathrm{~mm}$ resolution anatomically realistic model of the body induced by low frequency electric fields," Phys. Med. Biol., vol. 45, pp. 1013-1022, 2000.

[5] C. Gabriel, S. Gabriel, and E. Corthout, "The dielectric properties of biological tissues: I. Literature survey," Phys. Med. Biol., vol. 41, pp. 2231-2249, 1996.

[6] S. Gabriel, R. W. Lau, and C. Gabriel, "The dielectric properties of biological tissues: II. Measurements in the frequency range $10 \mathrm{~Hz}$ to 20 GHz," Phys. Med. Biol., vol. 41, p. 2251, 1996.
[7] S. Gabriel, R. W. Lau, and C. Gabriel, "The dielectric properties of biological tissues: III. Parametric models for the dielectric spectrum of tissues," Phys. Med. Biol., vol. 41, pp. 2271-2293, 1996.

[8] T. W. Dawson, K. Caputa, and M. A. Stuchly, "High-resolution organ dosimetry for human exposure to low-frequency electric fields," IEEE Trans. Power Del., vol. 13, no. 2, pp. 366-373, Apr. 1998.

[9] A. Barchanski, M. Clemens, H. De Gersem, and T. Weiland, "Efficient calculation of current densities in the human body induced by arbitrarily shaped, low-frequency magnetic field sources," J. Computat. Phys., vol. 214, no. 1, pp. 81-95, 2006.

[10] J. Dardenne, S. Valette, N. Siauve, N. Burais, and R. Prost, "Variational tetrahedral mesh generation from discrete volume data," Visual Comput., vol. 25, no. 5, pp. 401-410, 2009.

[11] B. F. Smith, P. Bjørstad, and W. Gropp, Domain Decomposition: Parallel Multilevel Methods for Elliptic Partial Differential Equations. Cambridge, U.K.: Cambridge Univ. Press, 2004.

[12] Y. Saad, Iterative Methods for Sparse Linear Systems. Warrendale, PA: Society for Industrial Mathematics, 2003.

[13] R. Scorretti, N. Burais, O. Fabrègue, A. Nicolas, and L. Nicolas, "Computation of the induced current density into the human body due to relative LF magnetic field generated by realistic devices," IEEE Trans. Magn., vol. 40, no. 2, pt. 2, pp. 643-646, Mar. 2004

[14] Y. Notay, "An aggregation-based algebraic multigrid method," ETNA, to be published.

[15] A. Toselli and O. B. Widlund, Domain Decomposition Methods-Algorithms and Theory. New York: Springer Verlag, 2005.

[16] Y. Notay, "Flexible conjugate gradients," SIAM J. Sci. Comput., vol. 22 , no. 4, pp. 1444-1460, 2000.

[17] W. T. Kaune, J. L. Guttman, and R. Kavet, "Comparison of coupling of humans to electric and magnetic fields with frequencies between 100 $\mathrm{Hz}$ and $100 \mathrm{kHz}$," Bioelectromagn., vol. 18, no. 1, pp. 67-76, 1998.

[18] K. Caputa, P. J. Dimbylow, T. W. Dawson, and M. A. Stuchly, "Modelling fields induced in humans by $50 / 60 \mathrm{~Hz}$ magnetic fields: Reliability of the results and effects of model variations," Phys. Med. Biol., vol. 47, pp. 1391-1398, 2002.

[19] L. H. Hoang, R. Scorretti, N. Burais, and D. Voyer, "Numerical dosimetry of induced phenomena in the human body by a three-phase power line," IEEE Trans. Magn., vol. 45, no. 3, pp. 1666-1669, Mar. 2009.

[20] P. J. Dimbylow, "Induced current densities from low-frequency magnetic fields in a $2 \mathrm{~mm}$ resolution, anatomically realistic model of the body," Phys. Med. Biol., vol. 43, pp. 221-230, 1998.

[21] M. J. Gander, "Optimized Schwarz methods," SIAM J. Numer. Anal. vol. 44, no. 2, pp. 699-731, 2006.

[22] A. Christ, W. Kainz, E. G. Hahn, K. Honegger, M. Zefferer, E. Neufeld, W. Rascher, R. Janka, W. Bautz, J. Chen, B. Kiefer, P. Schmitt, H.-P. Hollenbach, J. Shen, M. Oberle, D. Szczerba, A. Kam, J. W. Guag, and N. Kuster, "The virtual family-Development of surface-based anatomical models of two adults and two children for dosimetric simulations," Phys. Med. Biol., vol. 55, p. N23, 2010. 